\title{
Constraints on parity violation from the Cosmic Microwave Background
}

\section{Diego Molinari*}

Dipartimento di Fisica e Scienze della Terra, Università di Ferrara, Via Saragat 1, 44122

Ferrara, Italy

INFN, Sezione di Ferrara, Via Saragat 1, 44122 Ferrara, Italy

INAF-OAS Bologna, Istituto Nazionale di Astrofisica - Osservatorio di Astrofisica e Scienza dello Spazio di Bologna, Area della Ricerca del CNR, Via Gobetti 101, 40129, Bologna, Italy

E-mail: molinari@iasfbo.inaf.it

Parity violating extensions of the standard electromagnetism produce the cosmic birefringence effect, i.e. the in vacuo rotation of the linear polarization of the photons during their propagation. This effect, in its isotropic form, would generate a clear signature on the temperature and polarization anisotropies of the cosmic microwave background (CMB) and, in particular, it would switch on the TB and EB angular power spectra that otherwise would be null. We review the statistical methodologies developed to look for the isotropic birefringence effect and some recent results obtained using data from the ground based ACTpol experiment and from the Planck satellite. We also present forecasts on the ability of future CMB experiments to measure this effect.

Frontier Research in Astrophysics - III (FRAPWS2018)

28 May - 2 June 2018

Mondello (Palermo), Italy

* Speaker. 


\section{Introduction}

An interesting approach to test the fundamental laws of physics is to investigate phenomena that are not predicted by the standard models. Any signal that would confirm the existence of these phenomena may give hints for the presence of new models or more general theories that may extend the current ones.

One example is to look for a signal generated by parity violating processes that are not described by the standard model of particle physics. Several theoretical models have been already developed predicting a chiral nature of the gravitational field (see for example [1, 2]) or extensions of the Maxwell Lagrangian of electromagnetism including Chern-Simons type interactions in the electromagnetic Lagrangian [3], a quintessence field [4, 5], axion-like particles coupled with the electromagnetic field [6], spatial anisotropies during evolution of perturbations [7]. Here we will focus on the latter cases that predict a rotation of the photon polarization direction during propagation, an effect known as cosmic birefringence. At present days there has been several attempts to detect such a signal with all measurements compatible with no detection (see $[4,8,9]$ and references therein).

A linearly polarized photon may be always decomposed in a superposition of a left-handed and a right-handed circular polarization. In the simplest scenario, in the presence of a parity violating effect, the two circular polarizations will propagate with different velocities. As a consequence the polarization direction of the photon will rotate and the observer will measure it rotated by an angle called cosmic birefringence angle. Many astrophysical sources (see for example [10, 11, 12] have been investigated looking for parity violating processes. However, it is evident that if this birefringence effect is cumulative, the farther the source is, the larger will be the measured birefringence angle. The farthest source of linearly polarized photons is the Cosmic Microwave Background $(\mathrm{CMB})$ and therefore this radiation is a perfect test laboratory to constrain parity violating models.

The CMB temperature and polarization anisotropies are usually statistically described by expanding the fluctuations in spherical harmonics[13]. The coefficients of these functions are then used to build the so called Angular Power Spectra (APS). As predicted by the $\Lambda$ CDM model the fluctuations are Gaussian and therefore the Temperature (T) APS contains all the information about the temperature anisotropies. In polarization the signal may be decomposed in a gradient-like polarization patterns, or E-modes and a curl-like polarization patterns or B-modes. One important prediction of the $\Lambda \mathrm{CDM}$ model is that for parity conservation the correlations between temperature and B-modes and between E-modes and B-modes must be null. However, in the presence of an isotropic cosmic birefringence angle, $\alpha$, the APS that describe the CMB anisotropies are rotated and this rotation is parametrized as [2, 14]:

$$
\begin{aligned}
C_{\ell}^{\mathrm{TE}, \mathrm{obs}} & =C_{\ell}^{\mathrm{TE}} \cos (2 \alpha) \\
C_{\ell}^{\mathrm{TB}, \mathrm{obs}} & =C_{\ell}^{\mathrm{TE}} \sin (2 \alpha) \\
C_{\ell}^{\mathrm{EE}, \mathrm{obs}} & =C_{\ell}^{\mathrm{EE}} \cos ^{2}(2 \alpha)+C_{\ell}^{\mathrm{BB}} \sin ^{2}(2 \alpha) \\
C_{\ell}^{\mathrm{BB}, \mathrm{obs}} & =C_{\ell}^{\mathrm{BB}} \cos ^{2}(2 \alpha)+C_{\ell}^{\mathrm{EE}} \sin ^{2}(2 \alpha) \\
C_{\ell}^{\mathrm{EB}, \mathrm{obs}} & =\frac{1}{2}\left(C_{\ell}^{\mathrm{EE}}-C_{\ell}^{\mathrm{BB}}\right) \sin (4 \alpha)
\end{aligned}
$$


It is evident from Eq. 1.3 and 1.5 that the presence of parity violating processes will generate non null TB and EB correlations. An example of the kind of signal we may expect on TB and EB spectra in the presence of an isotropic birefringence angle is shown in Fig. 1 for different values of $\alpha$.
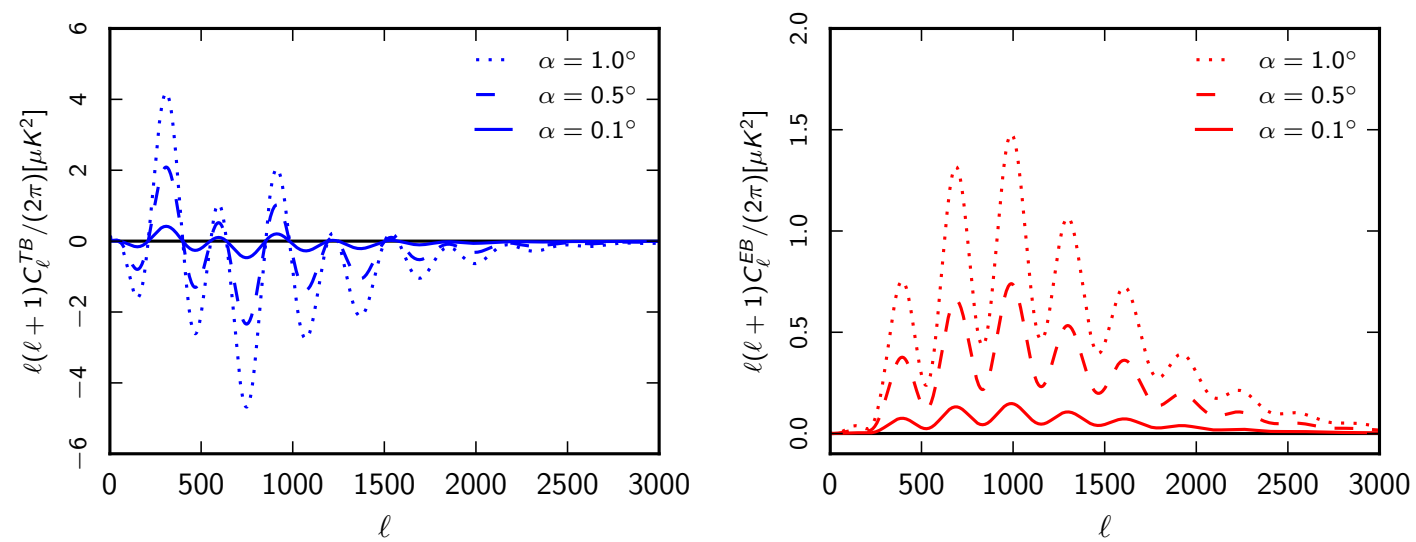

Figure 1: The expected oscillatory signal in TB and EB APS in the presence of an isotropic birefringence angle for several values of $\alpha$.

Recent CMB experiments derived very accurate measurements of the polarization anisotropy pattern allowing to look for the presence of non-zero TB and EB signals [15, 16, 17, 18]. In the following we briefly describe several approaches used in literature to estimate the amplitude of the isotropic birefringence angle. Focusing on one particular method we describe the results when it is applied in particular to CMB data from the Planck satellite [19] and from the ground-based ACTpol telescope [20]. We also describe forecasts for future CMB experiments showing that they will be able to reach an unprecedented accuracy in measuring $\alpha$. Finally we draw our conclusions.

\section{Methodologies}

Using cosmological data, a measurement of the cosmic birefringence angle, $\alpha$, may be done at least through one of the following methods [21]:

- Following a Monte Carlo Markov Chain (MCMC) approach through a likelihood coupled to an MCMC sampler to explore the space parameters of the $\Lambda$ CDM model extended to include the birefringence angle [22]. It is useful because it may investigate the correlations among the parameters. However, it needs a likelihood that includes all the 6 spectra and especially $\mathrm{TB}$ and $\mathrm{EB}$ to provide an accurate analysis.

- Applying a stacking analysis of polarization maps on temperature and E-mode extrema [23]. The former is sensitive to the amplitude of the TB APS, the latter to the amplitude of EB.

- Use the so called D-estimators [24] that involves a combination of the observed spectra:

$$
\begin{aligned}
& D_{\ell}^{\mathrm{TB}}(\tilde{\alpha})=C_{\ell}^{\mathrm{TB}, \mathrm{obs}} \cos (2 \tilde{\alpha})-C_{\ell}^{\mathrm{TE}, \mathrm{obs}} \sin (2 \tilde{\alpha}), \\
& D_{\ell}^{\mathrm{EB}}(\tilde{\alpha})=C_{\ell}^{\mathrm{EB}, \mathrm{obs}} \cos (4 \tilde{\alpha})-\frac{1}{2}\left(C_{\ell}^{\mathrm{EE}, \mathrm{obs}}-C_{\ell}^{\mathrm{BB}, \mathrm{obs}}\right) \sin (4 \tilde{\alpha}) .
\end{aligned}
$$



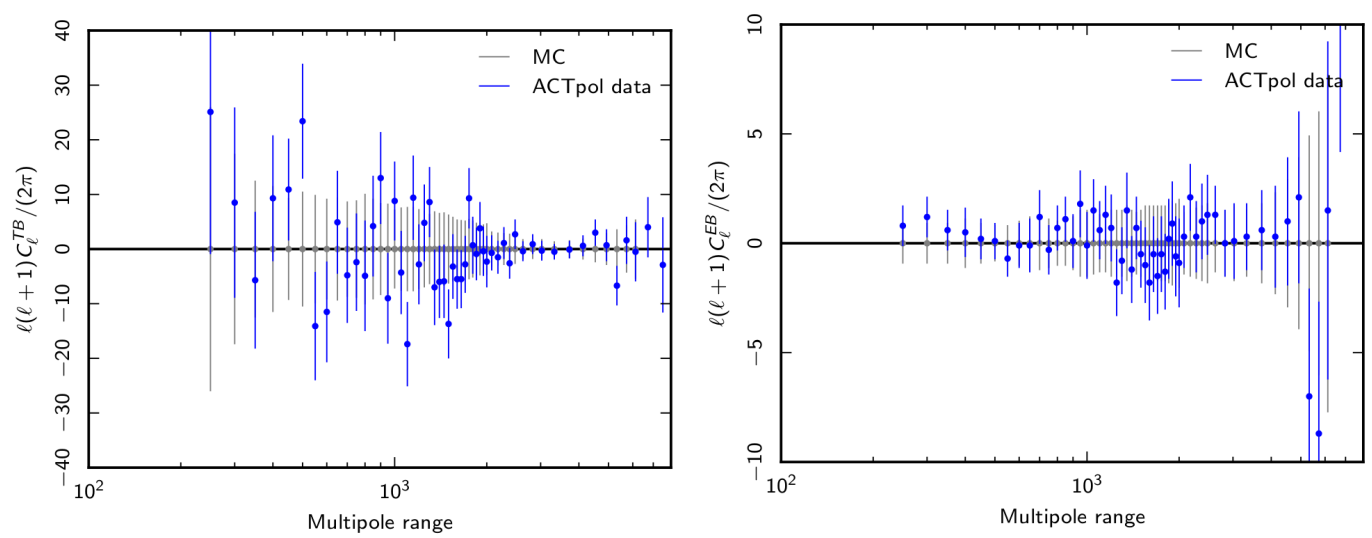

Figure 2: TB and EB spectra from ACTpol data [20]. Blue points are the real data, in grey we show the average and $1 \sigma$ dispersion of the realistic MC simulations we have generated internally for the analysis [25].

where $\hat{\alpha}$ is the estimated birefringence angle.

The three methods are complementary. In fact stacking is a pixel based analysis whilst Destimators work in harmonic space. Also in large missions like Planck the likelihood and the foreground cleaned maps pass through different data analysis pipelines. Therefore, when possible, it is important to apply all these methodologies on the same data to perform a stability check of the results.

The D-estimators are particularly interesting because have unique properties that allow for a deeper inspection of the available data. They depend explicitly on the multipole $\ell$ and therefore one can divide the multipole range observed by the instrument in different multipole intervals and look for the birefringence angle in each multipole. They use two different sets of APS allowing for a joint or an independent analysis of each one very useful for stability test. Also, the expectation value of the D-estimators is zero for each birefringence angle. It allows to look for $\alpha$ by finding the angle $\hat{\alpha}$ that minimizes the $\chi_{X}^{2}$ defined as:

$$
\chi_{X}^{2}(\tilde{\alpha})=\sum_{\ell \ell^{\prime}} D_{\ell}^{X} M_{\ell \ell^{\prime}}^{X X^{-1}} D_{\ell^{\prime}}^{X}
$$

where $X$ stands for either TB, EB or the two estimators jointly and the matrix $M_{\ell \ell^{\prime}}^{X X}=\left\langle D_{\ell}^{X} D_{\ell^{\prime}}^{X}\right\rangle$ is the covariance matrix of the considered D-estimator. In a frequentist approach the covariance matrix is built from MC simulations with no birefringence effect, but it is possible to use an analytic prescription to build a covariance matrix depending on $\alpha$ for a bayesian approach [21].

In the following we will focus on the D-estimators showing some application to recent data in a frequentist approach and forecasts for future experiments.

\section{Application to data}

In order to estimate the birefringence angle in the following examples a frequentist approach is considered. It assumes no detection and extracts an estimate of $\hat{\alpha}$ through eq. 2.3 from the data. The result is compared to the $\alpha$ distribution from a MC of simulations generated in the absence 
of birefringence effect. Any deviation or incompatibility between data and MC may be due to the presence of parity violating phenomena. In the following we show the results from the analysis of ACTpol data ${ }^{1}$ [25] and Planck data ${ }^{2}$ from the 2015 release [23].

\subsection{ACTpol data}

In Fig. 2 we show TB and EB spectra used to estimate the birefringence angle from ACTpol data [20].

In order to propagate the error through our pipeline, we generated a set of $10000 \mathrm{CMB}$ correlated APS with the same characteristics of the ACTpol data (for more details see [25]). The spectra are based on the Planck 2015 best fit model with the assumption of no birefringence effect. The MC average and dispersion are shown in gray in Fig. 2.

We compute the $\chi^{2}$ minimization of eq. 2.3 considering several multipole ranges and dividing the multipole range in intervals. In Fig. 3 we show the results of the analysis for $D_{\ell}^{T B}$ (blue points) or $D_{\ell}^{E B}$ (red points) or considering the two estimators jointly (green points).
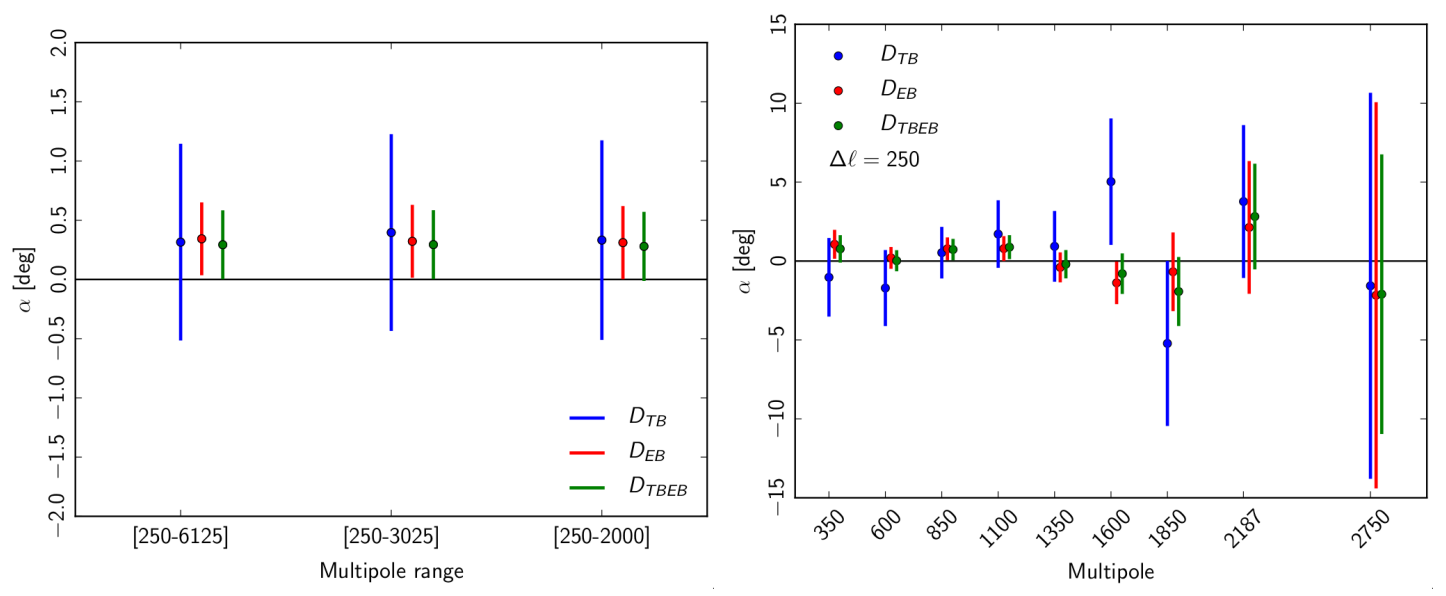

Figure 3: Birefringence angles estimated from ACTpol data considering different multipole ranges (left panel) and dividing the entire multipole range in intervals. In red we show the results considering only $D_{\ell}^{T B}$, in blue from $D_{\ell}^{E B}$ and in green the analysis considering the two estimators jointly [25].

All the results are compatible each other. We do not observe any effect on the results by changing the multipole range considered. When dividing the range in intervals the result in each interval is compatible with zero. The ACTpol data saturate the signal-to-noise ratio when considering the range $\ell=[250-3025]$. Therefore the best representative result from ACTpol data is $\hat{\alpha}=(0.29 \pm 0.28)^{\circ}$ that is compatible with no detection of a birefringence effect. In this result we show only the statistical error. However, we have to consider also a systematic error. In fact, the search for an isotropic birefringence angle is completely degenerate with a systematic effect induced by the uncertainty in the knowledge of the global orientation of the polarimeters of the focal plane. Therefore we need to add to the result a systematic error estimated to be $0.5^{\circ}$ [20].

\footnotetext{
${ }^{1} \mathrm{http} / / /$ lambda.gsfc.nasa.gov/product/act/actpol_prod_table.cfm

${ }^{2}$ pla.esac.esa.int/pla
} 


\subsection{Planck data}

In the analysis of Planck 2015 data we consider the component separated maps [19]. In Planck four different component separation algorithms are considered to remove the foreground contamination from the data: Commander, NILC, SMICA and SEVEM. In order to propagate the error induced by the cleaning procedure, each method passed through its pipeline a realistic MC of 1000 signal plus noise maps. In [23] we consider the products from all the four component separation pipelines for stability check. However, in order to minimize the noise contribution we consider only $D_{\ell}^{T B}$ that involves TB and TE spectra obtained by crossing temperature and polarization data that are supposed to have uncorrelated noise. In addition, in the 2015 release, SMICA provided cleaned data by dividing the time line of ordered data collected in two splits known as Half Mission. By crossing the two sets of maps it is possible to extract the APS removing the contribution of the uncorrelated noise. Only in this case we could safely consider an analysis involving the $D_{\ell}^{E B}$ estimator that uses EE and BB spectra that are more sensitive to autocorrelated noise or mismatch in the noise characterized by the simulations.

Using a pseudo-Cl code called Cromaster [26, 27, 28], we extract the APS from the cleaned data and MC simulations. In Fig. 4 we show the TB and EB APS extracted from Smica by crossing the Half Mission data. Although the $\Lambda$ CDM model used to generate the Planck MC simulations predicts TB and EB correlations to be null, realistic observations may cause non zero signals induced by the data analysis pipeline or by the presence of systematic effects. Realistic simulations should capture these effects to avoid biases in the analyses. This is why the Planck MC simulations (grey points) shown in Fig. 4 partially deviate from zero especially evident in TB APS.
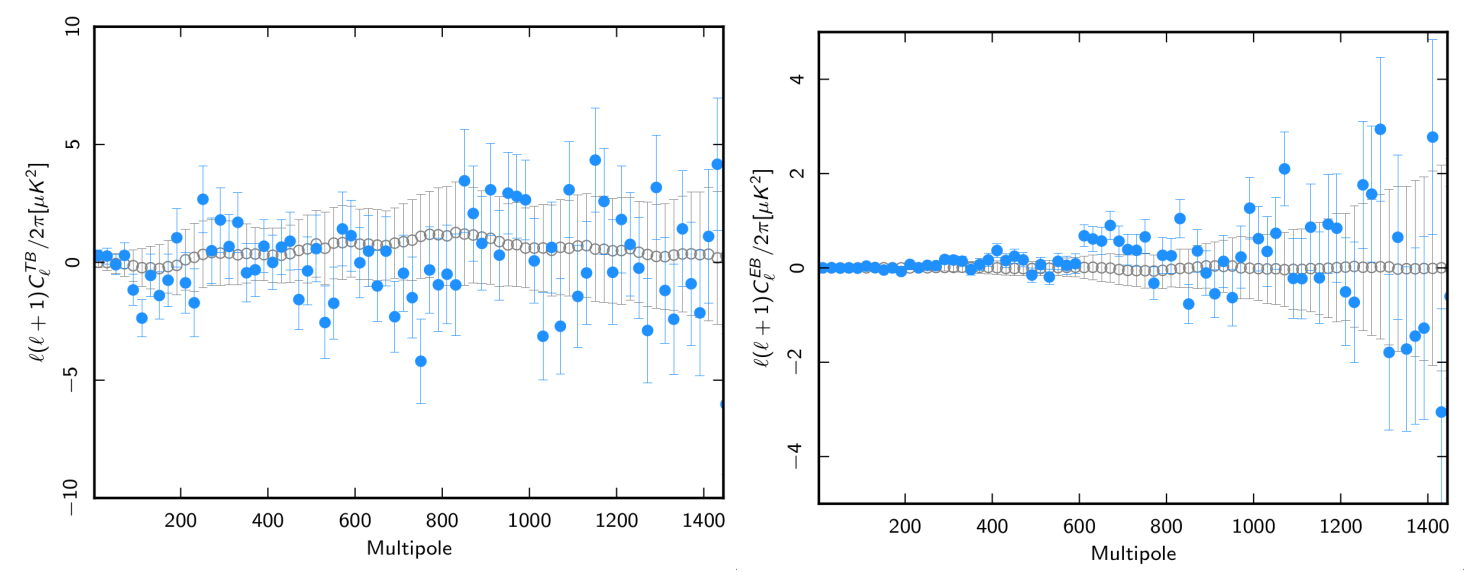

Figure 4: TB and EB APS from SMICA Half Mission component separated maps. In blue the real data, in grey the average and $1 \sigma$ dispersion of the realistic MC simulations [23].

Similarly to the ACTpol analysis we find the birefringence angle that minimizes the $\chi^{2}$ of eq. 2.3. The results are shown in Fig. 5. In the right panel we show the results when dividing the entire multipole range in intervals considering $D_{\ell}^{T B}$ (dots) or when possible $D_{\ell}^{E B}$ (stars) or the two estimators jointly (squares). In the left panel the results from the D-estimators (dots and stars) are compared to the results from the stacking analysis (squares). Different colors are related to different component separated maps considered. 

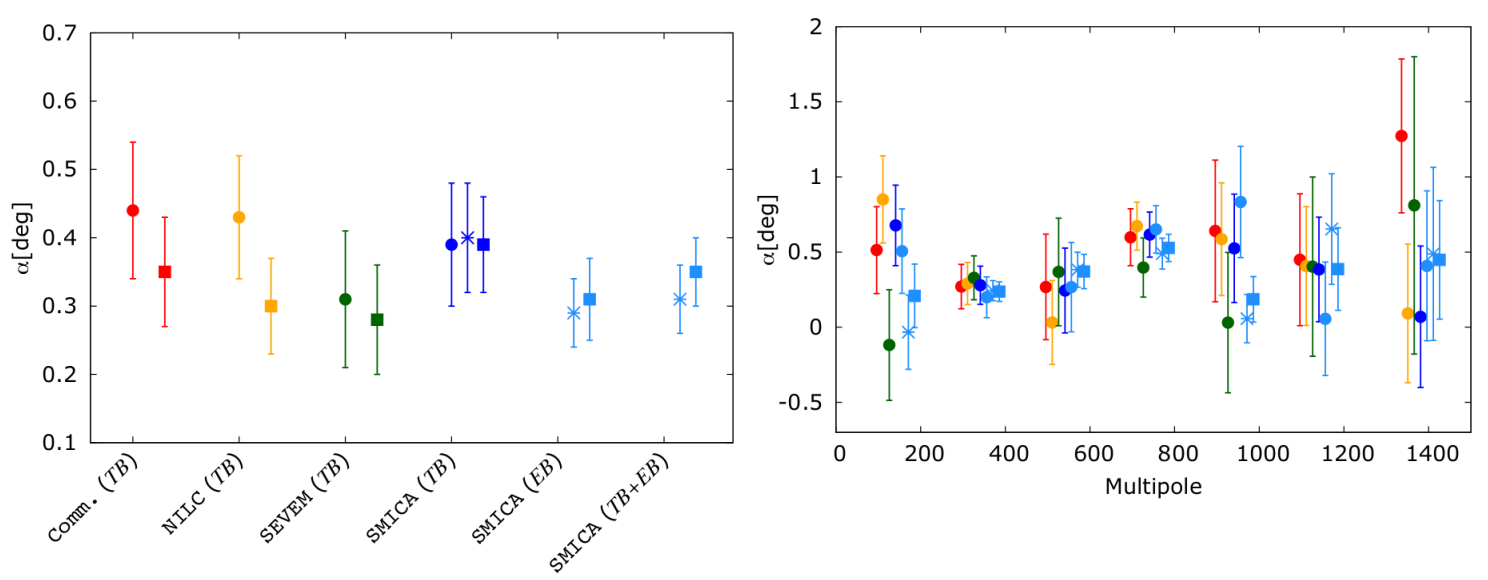

Figure 5: Birefringence angle estimated from Planck data considering different component separated products identified by different colors. In the left panel square points are relative to the results from the stacking analysis. In the right panel, where the entire multipole range is divided in intervals, dots are related to the results using $D_{\ell}^{T B}$, star from using $D_{\ell}^{E B}$ and squares from the joint use of $D_{\ell}^{T B}$ and $D_{\ell}^{E B}$. Only the statistical error is shown [23].

The results are very consistent each other. As expected the results using $D_{\ell}^{E B}$ are more accurate providing smaller error bars. Nonetheless all the estimates among the component separated maps considered and the different channels are compatible with each other either when considering the entire multipole range or when dividing it in intervals. Also the results from D-estimators are very consistent with the results from the stacking analysis. The best representative result is obtained considering SMICA Half Mission data with the joint channel and it is $6 \sigma$ far from the null effect. However, when we include an estimate of the systematic error induced by the uncertainty in the polarimeter orientation [29] the final result from Planck data is $\hat{\alpha}=(0.31 \pm 0.05 \pm 0.3)^{\circ}$ compatible with no detection of a birefringence effect. It is anyway interesting to note that the error budget in this Planck data analysis is dominated by the systematic uncertainty.

In Fig. 6 all the birefringence angle constraints from CMB data are summarized [23]. Up to date all the experiments obtained results compatible with no detection.

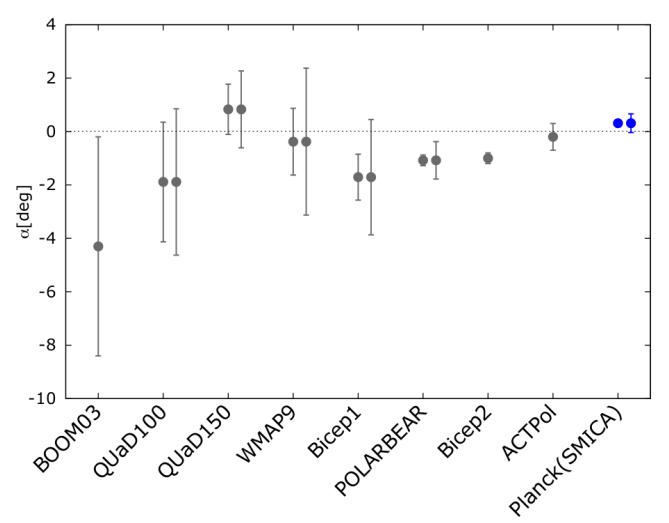

Figure 6: Summary of the birefringence angle measurements from several CMB experiments from BOOMERaNG to Planck ([23] adapted from [30] in gray). 


\section{Forecasts}

The methodology developed to generate MC APS simulations for the ACTpol analysis may be used to provide forecasts for future experiments [25]. We considered the characteristics of few representative CMB experiments including ground based telescopes (Advanced ACTpol [31]), balloon borne experiments (LSPE [32]), and satellite missions (LiteBIRD [33] and COrE [34]). Implementing the characteristics of the proposed experiments and generating the MC APS simulations, we derive the accuracy in the estimation of the birefringence angle, shown in Fig. 7, using $D_{\ell}^{T B}$ and $D_{\ell}^{E B}$ estimators obtained by measuring the dispersion of the $\alpha$ angles obtained from the MC simulations.

It is important to note that these forecasts consider only the limitation to the accuracy due to white noise. We do not consider possible systematic effects that may be very different among the experiments considered. In addition, the forecasts provided are valid only if in the post-processing of the data collected by these experiments they are absolutely calibrated and the so called EBnulling technique is not applied. This technique rotates $\mathrm{Q}$ and $\mathrm{U}$ maps in order to have a null EB APS assuming that any deviation from zero in EB is totally due to a mis-orientation of the polarimeters. It is clear how this technique totally prevents us from the study of an isotropic birefringence effect.

Forecasts show that thanks to both a larger sky coverage and a better resolution than LiteBIRD, the COrE-like satellite provides the best accuracy in measuring the birefringence angle. It will be able to detect $\alpha$ with a $1 \sigma$ error of the order of $10 \operatorname{arcsec}$. This is more than one order of magnitude better than the statistical error of the actual Planck data. This is also 4 times better than what expected from LiteBIRD data due to the different resolution. Advanced ACTpol experiment will achieve a sensitivity which is intermediate between LiteBIRD and COrE. Although LSPE has the lowest resolution among the cases considered, in the case of no systematic effects it will achieve bounds on $\alpha$ about twice as stringent as present day high resolution experiments.

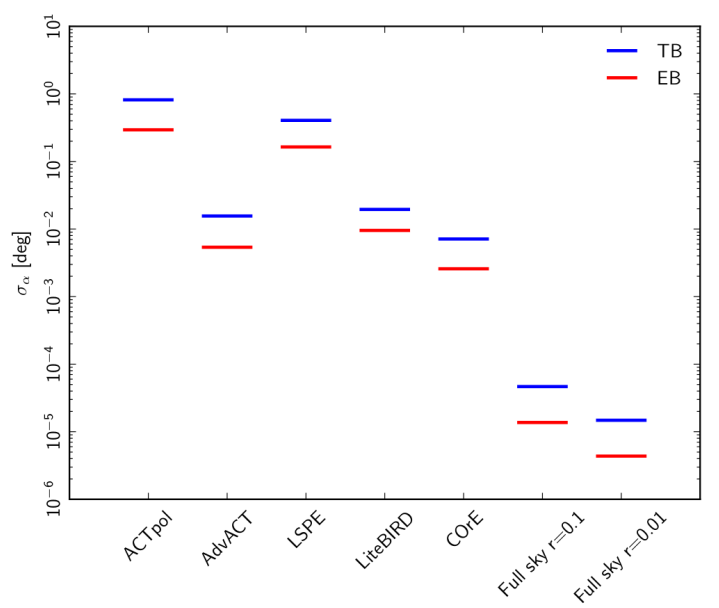

Figure 7: Forecasts for $1 \sigma$ errors in the measurement of the birefringence angle for the future experiments listed. We show forecasts for $D_{\ell}^{T B}$ (blue lines) and $D_{\ell}^{E B}$ (red line) estimators. For comparison we show the results from ACTpol measurements described above and two ideal cosmic variance limited cases with $\mathrm{r}=0.1$ and $0.01[25]$. 


\section{Conclusions}

Some theories beyond the standard model of particle physics predict the presence of parity violating phenomena. They would rotate in vacuo the photon polarization direction during propagation, an effect called cosmic birefringence. CMB radiation is particularly useful to test these models because the rotation would leave specific footprints in the temperature and polarization anisotropies generating TB and EB spectra otherwise expected to be null in the $\Lambda$ CDM model.

We have described some methodologies to investigate for the presence of these footprints and, focusing on the so-called D-estimators, we have shown their application to ACTpol data and Planck data looking for an isotropic birefringence effect. The results were able to constrain the amplitude of the birefringence angle to be $\hat{\alpha}=(0.29 \pm 0.28 \pm 0.5)^{\circ}$ for ACTpol and $\hat{\alpha}=(0.31 \pm 0.05 \pm 0.3)^{\circ}$ for Planck. Here we included an estimate of the statistical error and of the systematic error related to the degeneracy of the effect with the uncertainty in the orientation of the polarimeters of the focal plane. Once including the systematic error both the estimates are compatible with no detection. It is important to highlight that the error budget of the Planck results is dominated by the systematic effects.

We also developed a methodology to provide forecasts for future CMB observations showing the expected sensitivity in measuring the birefringence angle. We explored examples of a ground based telescope, Advanced ACTpol, a balloon borne experiment, LSPE and two satellite missions, LiteBIRD and COrE. Considering their white noise limit, the resolution and the sky coverage we provided forecasts showing that COrE will be able to measure a birefringence angle with a $1 \sigma$ sensitivity of $10 \mathrm{arcsec}$, more than one order of magnitude better than the actual constraints.

The results shown in this work are all related to the search for an isotropic birefringence angle, but many models predict spatial fluctuations of the birefringence effect and therefore the footprint of such an effect may be investigated not only in its monopole, but in its entire angular power spectrum. The extraction of the APS of the birefringence angle may allow to deeper investigate the effect and further help in distinguishing among the many models suggested. Already some analyses in this perspective has been considered using data from WMAP [35], POLARBEAR [36] or BICEP2 [37].

The search for a cosmic birefringence effect may shed a light on new physics beyond the standard model of particles. Future CMB observations will be able to dramatically improve the accuracy in this kind of analyses.

\section{References}

[1] B. Thorne, T. Fujita, M. Hazumi, N. Katayama, E. Komatsu and M. Shiraishi, Phys. Rev. D 97 (2018) no.4, 043506

[2] A. Lue, L. M. Wang and M. Kamionkowski, Phys. Rev. Lett. 83 (1999) 1506

[3] S. M. Carroll, G. B. Field and R. Jackiw, Phys. Rev. D 41 (1990) 1231.

[4] S. M. Carroll, Phys. Rev. Lett. 81 (1998) 3067

[5] M. Giovannini, Phys. Rev. D 71 (2005) 021301

[6] F. Finelli and M. Galaverni, Phys. Rev. D 79 (2009) 063002 
[7] N. Bartolo, S. Matarrese, M. Peloso and M. Shiraishi, JCAP 1507 (2015) no.07, 039

[8] S. di Serego Alighieri, F. Finelli and M. Galaverni, Astrophys. J. 715 (2010) 33

[9] M. Kamionkowski, Phys. Rev. D 82 (2010) 047302

[10] A. Cimatti, S. di Serego Alighieri, G. B. Field and R. A. E. Fosbury, Astrophys. J. 422 (1994) 562.

[11] L. Maccione, S. Liberati, A. Celotti, J. G. Kirk and P. Ubertini, Phys. Rev. D 78 (2008) 103003

[12] Y. Z. Fan, D. M. Wei and D. Xu, Mon. Not. Roy. Astron. Soc. 376 (2006) 1857

[13] M. Kamionkowski, A. Kosowsky and A. Stebbins, Phys. Rev. D 55 (1997) 7368

[14] B. Feng, H. Li, M. z. Li and X. m. Zhang, Phys. Lett. B 620 (2005) 27

[15] Planck Collaboration, Astron. Astrophys. 594 (2016) A1

[16] BICEP2 and Planck Collaborations, Phys. Rev. Lett. 114 (2015) 101301

[17] J. L. Sievers et al. [Atacama Cosmology Telescope Collaboration], JCAP 1310 (2013) 060

[18] K. K. Schaffer et al., Astrophys. J. 743 (2011) 90

[19] Planck Collaboration, Astron. Astrophys. 594 (2016) A9

[20] S. Naess et al. [ACTPol Collaboration], JCAP 1410 (2014) no.10, 007

[21] A. Gruppuso, G. Maggio, D. Molinari and P. Natoli, JCAP 1605 (2016) no.05, 020

[22] A. Gruppuso, M. Gerbino, P. Natoli, L. Pagano, N. Mandolesi, A. Melchiorri and D. Molinari, JCAP 1606 (2016) no.06, 001

[23] Planck Collaboration, Astron. Astrophys. 596 (2016) A110

[24] E. Y. S. Wu et al. [QUaD Collaboration], Phys. Rev. Lett. 102 (2009) 161302

[25] D. Molinari, A. Gruppuso and P. Natoli, Phys. Dark Univ. 14 (2016) 65

[26] E. Hivon, K. M. Gorski, C. B. Netterfield, B. P. Crill, S. Prunet and F. Hansen, Astrophys. J. 567 (2002) 2

[27] G. Polenta, D. Marinucci, A. Balbi, P. de Bernardis, E. Hivon, S. Masi, P. Natoli and N. Vittorio, JCAP 0511 (2005) 001

[28] D. Molinari, A. Gruppuso, G. Polenta, C. Burigana, A. De Rosa, P. Natoli, F. Finelli and F. Paci, Mon. Not. Roy. Astron. Soc. 440 (2014) no.2, 957

[29] C. Rosset et al., Astron. Astrophys. 520 (2010) A13

[30] J. P. Kaufman, B. G. Keating and B. R. Johnson, Mon. Not. Roy. Astron. Soc. 455 (2016) no.2, 1981

[31] S. W. Henderson et al., J. Low. Temp. Phys. 184 (2016) no.3-4, 772

[32] S. Aiola et al. [LSPE Collaboration], Proc. SPIE Int. Soc. Opt. Eng. 8446 (2012) 84467A

[33] T. Matsumura et al., J. Low. Temp. Phys. 176 (2014) 733

[34] J. Delabrouille et al. [CORE Collaboration], JCAP 1804 (2018) no.04, 014

[35] V. Gluscevic, D. Hanson, M. Kamionkowski and C. M. Hirata, Phys. Rev. D 86 (2012) 103529

[36] POLARBEAR Collaboration, Phys. Rev. D 92 (2015) 123509

[37] BICEP2 and Keck Arrary Collaborations, Phys. Rev. D 96 (2017) no.10, 102003 


\section{DISCUSSION}

GIULIO AURIEMMA: I wonder if it is possible to detect CP violations in the CMBR interaction with baryons.

DIEGO ANSWER: As far as I know, the CMB photons in the standard cosmological model interact with the charged baryons of the plasma through Thomson scattering. In the model the interaction is the same if the baryon is composed by matter or antimatter. What really matters in the evolution of perturbations is the local density of the baryons. Therefore I think that $\mathrm{CP}$ violations cannot be directly observed looking at CMB photons. However, baryogenesis needs some violations of the standard model of particle physics. It may be that indirectly CMB data will help in understanding what mechanism happened during the baryogenesis. 